\title{
Simulation of NMR response from micro-CT images using artificial neural networks
}

\author{
Reza Farzi ${ }^{1}$, Vahid Bolandi ${ }^{2}$, Ali Kadkhodaie ${ }^{3,4}$, Stefan Iglauer $^{3}$, Zeinolaabedin Hashempour ${ }^{1}$ \\ 1. School of Geology, University College of Science, University of Tehran, Tehran, Iran \\ 2. Department of Geology, Faculty of Earth Sciences, S. Chamran University of Ahvaz, Ahvaz, Iran \\ 3. Department of Petroleum Engineering, Curtin University, Perth, WA. \\ 4. Geology Department, Faculty of Natural Science, University of Tabriz, Tabriz, NW Iran, Iran
}

\begin{abstract}
The Nuclear Magnetic Resonance (NMR) log is amongst the functional techniques in petroleum investigation to segregating the reservoir and non-reservoir horizons precisely; furthermore, the NMR log provides an improved method to determine reservoir petrophysical parameters. Unfortunately, these data are usually sparse since acquiring NMR logs in producing cased wells is not possible and it is one of the most expensive tools in the logging industry thus its associated costs are the major limitation of its usage. Consequently, researchers have recently studied to virtually extract the NMR parameters via other routes. One such route, which we propose here is the possibility of estimating the $T_{2}$ distribution curve and magnetization decay by establishing a relationship between micro-CT images and NMR parameters by means of artificial neural networks (ANN) and image analysis algorithms. Specifically, two ANN networks were designed, which numerically image features from micro-CT images as inputs, while the amplitude of the magnetization and relaxation time were output parameters. We assessed the procedure by taking the error rate and correlation coefficient into consideration and we conclude that the ANN model is capable of finding logical patterns between image features and NMR responses, and is thus able to reliably predict NMR response behavior. Furthermore, we quantitatively compared ANN and random walk (RW) NMR predictions, and we demonstrate that ANN readily outperforms RW in terms of accuracy.
\end{abstract}




\section{Introduction}

The quality of a reservoir system controls the economic profitability of a field. Typically this quality is assessed by conventional porosity and resistivity logs, however these logs have serious limitations (Asquith and Krygowski, 2004); for example the presence of clay leads to errors in porosity estimation, since porosity $\operatorname{logs}$ (neutron, density and sonic) strongly relies upon rock matrix than reservoir fluids properties (Asquith and Krygowski, 2004). Furthermore, the resistivity tools are unable to distinguish between clay-bound, capillary-bound and reservoir's moveable water, which ends in an overestimation of the amount of water saturation and ignoring the reservoir units in some cases. These limitations were tackled by NMR logging (which introduced in 1978 by Schlumberger (Maute, 1992)), which played an important role in the specification of reservoir pore architecture and characterization and measurement of oil and gas in the formation. NMR logging is non-destructive, continuous and fast, which is certainly superior to conventional methods, providing reservoir appraisement without the environmental impacts possible (Coates et al., 1999). Technically the NMR measurement responds only to hydrogen nuclei (in water, oil and gas) in the pore space (Darling, 2005). For this reason, NMR logging provides the feasibility of immediate quantification of porosity and the differentiation of fluid types and other important petrophysical parameters being connected to the pore morphology (Darling, 2005).

Since NMR logging is one of the most expensive tools in the logging industry (Blümich, 2005), researchers have recently studied to virtually extract the NMR parameters via different routes. Up to now, most studies focused on the exploitation of Artificial intelligence (AI) as an estimator to simulate the NMR parameters from conventional well log data, e.g. Mohaghegh et al. (2001) applied AI methods to predict the NMR-derived permeability, effective porosity and irreducible water saturation in a field located in East Texas; while Labani et al. (2010) struggled to estimate the NMR free fluid porosity and permeability from well log data using AI. Golsanami et al. (2014) used fuzzy logic, neuro-fuzzy and neural networks to estimate the magnetic resonance $T_{2}$ distribution and the $T_{2}$ logarithmic mean from well $\log$ data in the Zagros Basin, Iran. Furthermore, Talabi et al. (2009) simulated $\mathrm{T}_{2}$ distribution and 
magnetization decay by establishing a relationship between micro-CT images and NMR parameters using a random walk (RW) method, this stochastic process consists of successive random steps (Lawler and Limic, 2010). Talabi et al. (2009) synthesized the NMR parameters using random walk theory in which the pore space voxels of sand packs were successively surveyed. The current study proposes a new numerical procedure for estimation of the $\mathrm{T}_{2}$ curve and magnetization decay from micro-CT images integrating image analysis and neural networks. The chief section of the suggested process is a voxelbased feature extraction as a branch of image analysis techniques. The main steps of the methodology are shown in Fig. 1. The reliability of the technique is verified by the application of the samples displayed in Talabi et al. (2009).

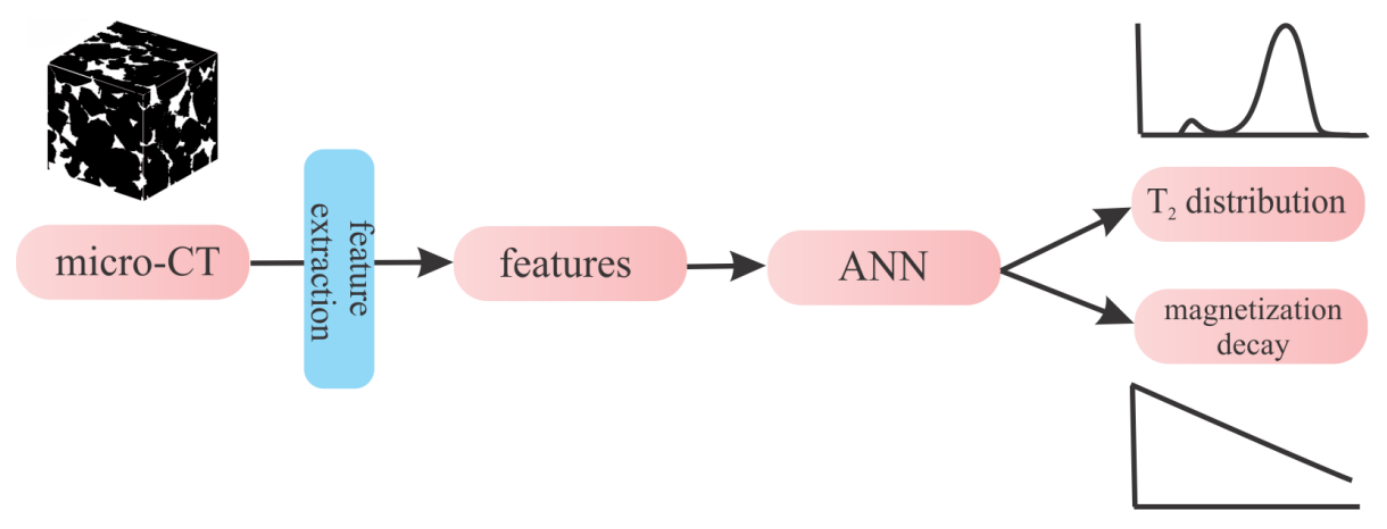

Fig. 1. Flowchart of proposed methodology.

\section{Theoretical background}

\subsection{NMR data}

The NMR log uses a radio frequency $(\mathrm{RF})$ transmitter, receiver, and a permanent magnet. Before the tool generates magnetic fields that align the spin axes of the protons in a specific route, hydrogen protons pertaining to formation fluids was found to have random orientation throughout the pore space. To generate a measurable signal, the polarized protons must be at a condition of resonance. Resonance is achieved by pulsing the formation with an oscillating field to force these protons out of their new equilibrium condition. Once the oscillatory magnetic field is taken away, the magnetic signal emitted by 
spinning protons relaxing toward the original condition. These protons generate a series of so-called spin echoes by applying a sequence of repetitive pulses with the NMR tool. To synthesize a spin-echo train (which constitutes the raw NMR data), an NMR tool measures the amplitude of the spin echoes as a function of time.

The (transverse magnetization) amplitude of the spin-echo train at time $t$ is given by (Kenyon, 1997)

$M_{x}(t)=M_{0 x} e^{\frac{-t}{T_{2}}}$

Wherein the equivalent magnitude of the transverse magnetization at $\mathrm{t}=0$ is $M_{0 x}, T_{2}$ is the time constant of the transverse magnetization decay (the transverse relaxation time).

Determining the $\mathrm{T} 2$ distribution is of paramount importance in NMR data analysis. This step is a mathematical inversion process called echo-fit or mapping. Due to the $\mathrm{T} 2$ distribution of rock being a continuous function, a multi-exponential model is being utilized by the mapping process that assumes that the $\mathrm{T} 2$ distribution comprises of $\mathrm{m}$ discrete relaxation times $\mathrm{T} 2 \mathrm{i}$ with corresponding porosity components фi. Eq. 2 displays the system of equations that present the individual echoes (Coates et al., 1999).

$$
\begin{aligned}
& \operatorname{echo}(1)=\phi_{1} e^{-\left[t(1) / T_{2,1}\right]}+\phi_{2} e^{\left.-f^{t(1)} / T_{2,2}\right]}+\phi_{3} e^{-\left[t(1) / T_{2,3}\right]}+\cdots+\phi_{m} e^{-\left[t(1) / T_{2, m}\right]}+\text { noise } \\
& \operatorname{echo(2)}=\phi_{1} e^{-\left[t(2) / T_{2,1}\right]}+\phi_{2} e^{-\left[t(2) / T_{2,2}\right]}+\phi_{3} e^{-\left[t(2) / T_{2,3}\right]}+\cdots+\phi_{m} e^{-\left[t(2) / T_{2, m}\right]}+\text { noise } \\
& \vdots \\
& \operatorname{echo}(n)=\phi_{1} e^{-\left[t(n) / T_{2,1}\right]}+\phi_{2} e^{-\left[t(n) / T_{2,2}\right]}+\phi_{3} e^{-\left[t(n) / T_{2,3}\right]}+\cdots+\phi_{m} e^{-\left[t(n) / T_{2, m}\right]}+\text { noise }
\end{aligned}
$$

Where through $t(i)$ is the pertaining time as the ith echo was obtained. 


\subsection{Image features extraction}

In brief, Feature extraction is a low-level image analysis operation and composed of a sequence of operations on the image matrix (Marmo et al., 2005). An image feature standardly is a part of an image that encompasses fascinating attributes or a property of the image which we are interested in, depending on the overall goal and what the application is trying to achieve. Correspondingly, in this study several tests have been performed on micro-CT images prepared in binary mode (black voxels are the grains and white voxels represent pore space) to acquire 21 features (f1-f21) stated in Marmo et al. (2005) as bellow:

$\mathrm{f} 1$ = ratio of edge voxels to the whole voxel;

$\mathrm{f} 2=$ number of white areas;

f3 = number of voxel of white areas;

$\mathrm{f} 4=$ number of white areas composed of more than 150 voxels;

f5 = number of voxels within the white areas composed of more than 150 voxels;

These previous five features (1-5) were extracted from the whole image, while the 16 remaining features were obtained out of the each four largest selected white areas.

$\mathrm{f6}-\mathrm{f} 9=$ number of voxel in the white area;

$\mathrm{f} 10-\mathrm{f} 13=$ length in voxel of the white area;

$\mathrm{f} 14-\mathrm{f} 21=$ number of small and large convex deficiencies;

The smallest convex polygon is called convex hull, embodying the binary object which the corresponding convex deficiency are computed as important features (Liu-Yu et al., 1997). A labeling algorithm detects and quantifies the convex deficiencies. The values 100 and 1000 are cut off criteria for a number of 
voxels to detect convex deficiencies. Hence, the large and small convex deficiencies were determined with more than 1000 voxels and less than 100 voxels, respectively.

Fig. 2 displays the disparity between the features for two samples of sandstone and carbonate rock with different sedimentological and petrophysical parameters. 

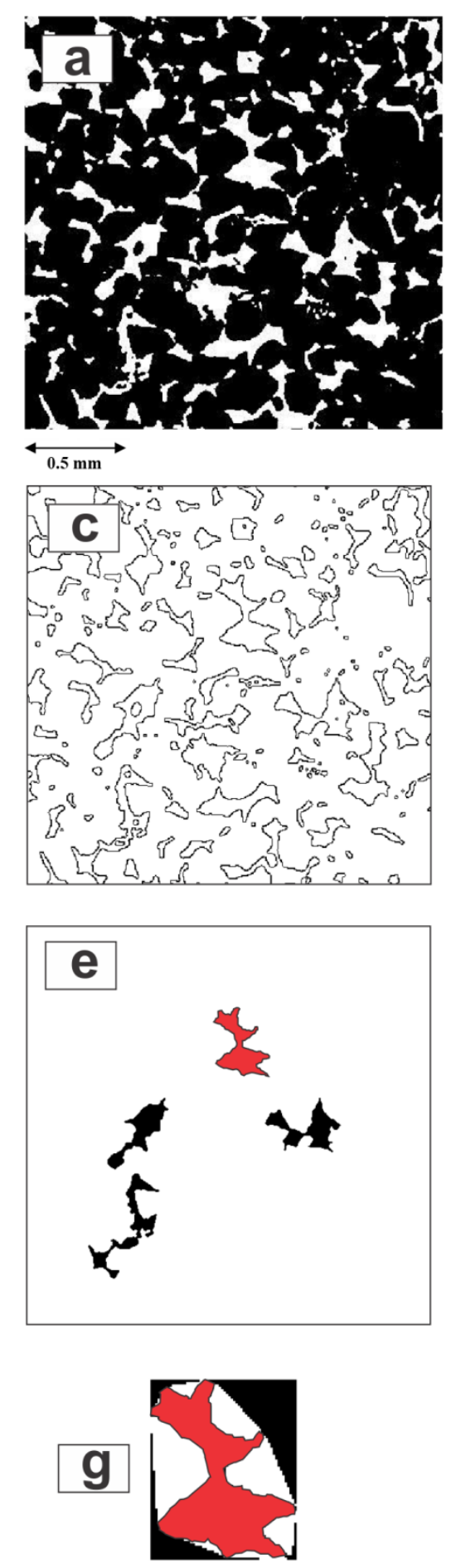

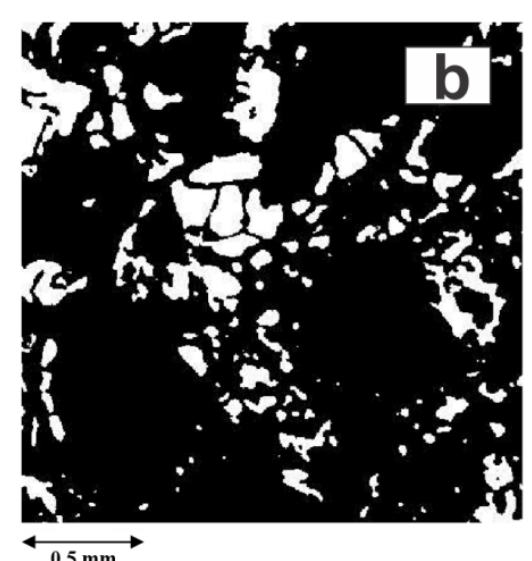

$\underset{0.5 \mathrm{~mm}}{\longrightarrow}$
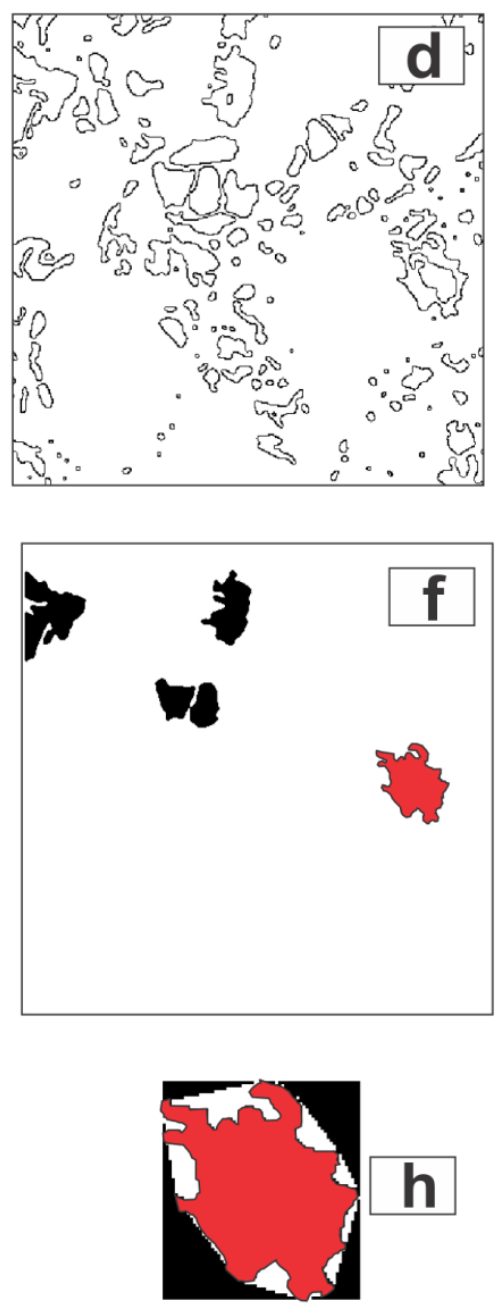

Fig. 2. (a) Binary micro-CT image slice through a sandstone (white voxels show pore space), (b) Binary micro-CT image slice through a carbonate rock (white voxels show pore space), (c-d) Edge voxels of a and b, (e-f) four selected white areas with more than 150 voxels from c and d, (g-f) Convex deficiency of one of the selected area in e and $\mathrm{f}$. 


\section{Results and discussion}

\subsection{Sample preparation}

In the current investigation, previously achieved data from the samples displayed in Talabi (2008) and Talabi et al. (2009) was analyzed. In summary, eight samples (six sand packs (F42A, F42B, F42C, LV60A, LV60B and LV60C) and two sandstones (Berea and S3)) were selected for micro-CT imaging and NMR measurements. To determining the grain size distributions of the sands British standard meshes were used on an electric shaker sieving the samples for 80 min (Talabi, 2008). The sands were then poured into a $10 \mathrm{~cm}$ long thermoplastic heat shrink sleeve (diameter $3.81 \mathrm{~cm}$ ) fitted with plastic end caps and pieces of circular filter paper to ensure a tight fit and so that no sand was lost. An electric vibrator used for tapping and vibrating to ensure compaction, then the samples were placed in an oven at $80{ }^{\circ} \mathrm{C}$ for $20 \mathrm{~min}$ and then the samples were left to cool (Talabi et al., 2009).

\subsection{NMR measurements}

Brine saturated sand packs (3.81 cm in diameter by $10 \mathrm{~cm}$ in length) were used for the NMR measurement. The brine was a solution of de-ionized water with 5 wt. \% Sodium Chloride ( $\mathrm{NaCl})$ and 1 wt. \% Potassium Chloride $(\mathrm{KCl})$. The reason for adding $\mathrm{NaCl}$ was to increase the ionic strength of the water (to better approximate formation brine) and $\mathrm{KCl}$ was used to prevent the sand from swelling (clay stabilization). NMR relaxation measurements were then performed on a MARAN2 bench top spectrometer at a temperature of $308 \mathrm{~K}$ and at $2 \mathrm{MHz}$. The magnetization decay ( $2 \mu$ s inter-echo spacing) then generated the $T_{2}$ relaxation time distribution applying a curvature-smoothing regularization method (Chen et al., 1999; Toumelin et al., 2003).

\subsection{Micro-CT imaging}

The micro-CT images (the samples had following dimensions: $0.65 \mathrm{~cm}$ diameter and length of $4 \mathrm{~cm}$ ) were acquired on a commercial XMT unit (Phoenix-X-ray System and Services GmbH). These greyscale micro-CT images were then filtered and converted to a binary format with the purpose of simulating the 
NMR parameters. Binarized 3D micro-CT images of F42A-C and LV60A-C sand packs and Berea and S3 sandstones are illustrated in Fig. 3 (also compare Talabi et al. 2008).
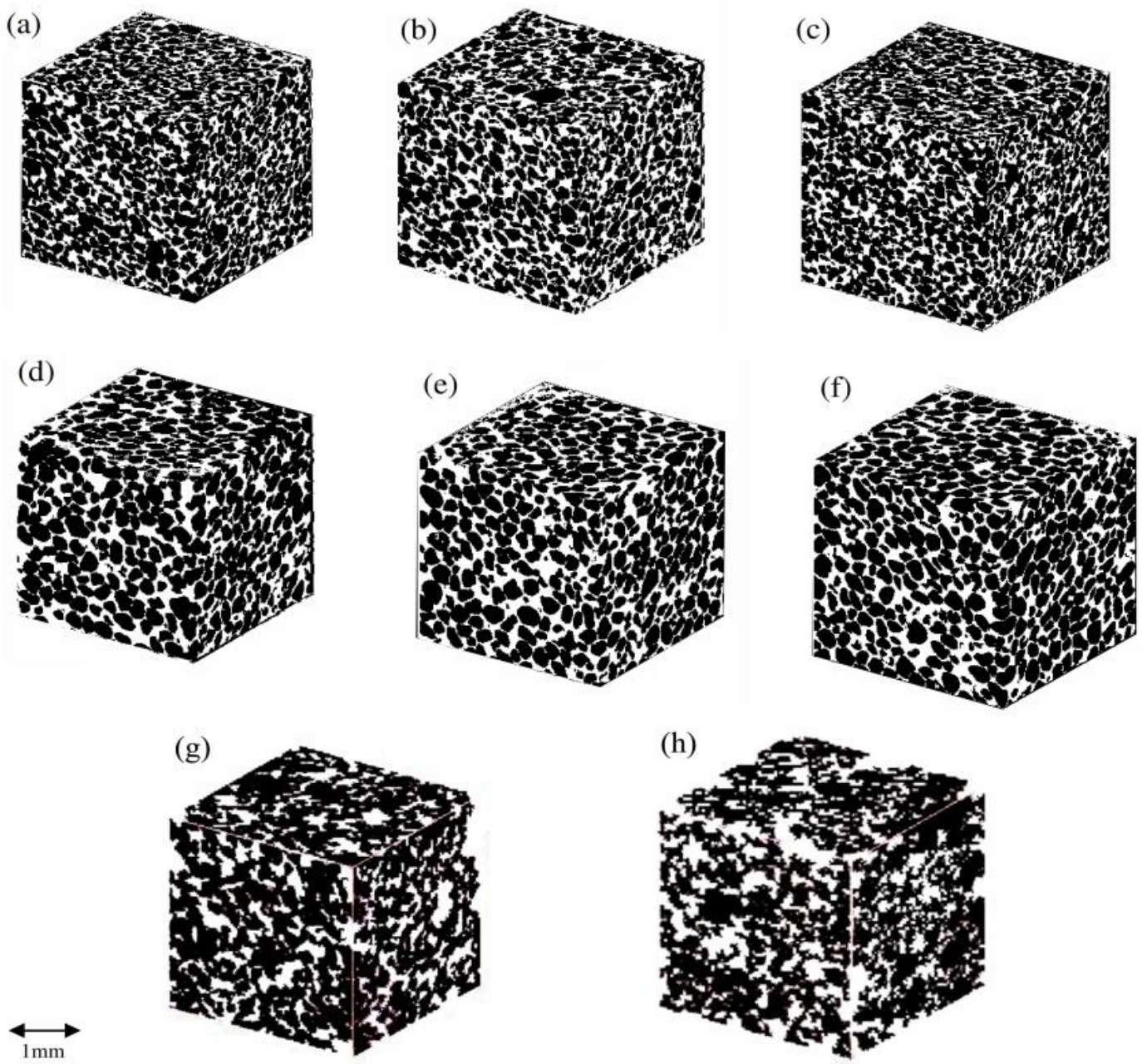

Fig. 3. Binarized 3D micro-CT images of (a) LV60A, (b) LV60B, (c) LV60C, (d) F42A, (e) F42B, (f) F42C, (g) Berea and (h) S3.

\subsection{Image features extraction}

The image analysis techniques allow conversion of an object into a set of numbers, i.e., some information extraction; the outcome of image analysis is a number or a bunch of numbers or a graph, it is not an image (Heilbronner and Barrett, 2014). This methodology was utilized on the binarized images, created databases were prepared as input parameters into the ANN model. Accordingly, scanned images of $750 \times$ 
$750 \times 450$ voxels, with resolutions ranging from 8 to $10 \mu \mathrm{m}$ were collected and analyzed in MATLAB ${ }^{\circledR}$. Table 1 illustrates the numerical values of aforementioned features of each sample.

An essential issue that should be considered is rock heterogeneity. A significant size difference between samples used for NMR measurement and micro-CT images leads to uncertainty. In order to avoid its effect on the results, the scale of the NMR measurements and micro-CT imaging must be similar.

Table 1. Numerical image features of micro-CT images.

\begin{tabular}{|c|c|c|c|c|c|c|c|c|}
\hline & F42A & F42B & F42C & LV60A & LV60B & LV60C & Berea & S3 \\
\hline f1 & 0.08422962 & 0.08889211 & 0.09030005 & 0.11858438 & 0.10871405 & 0.12689751 & 0.07270977 & 0.08815466 \\
\hline $\mathbf{f 2}$ & 1054 & 819 & 610 & 537 & 515 & 1037 & 7578 & 5807 \\
\hline $\mathbf{f 3}$ & 9144359 & 11002886 & 11381890 & 13668674 & 13549403 & 11775246 & 4388720 & 1780428 \\
\hline f4 & 357 & 174 & 167 & 69 & 97 & 211 & 1071 & 73 \\
\hline f5 & 8859557 & 10766833 & 11211463 & 13512938 & 13404436 & 11484926 & 1853851 & 97976 \\
\hline f6 & 716090 & 2570125 & 1470676 & 3092727 & 1807632 & 575299 & 5204 & 2353 \\
\hline $\mathbf{f} 7$ & 443154 & 1976595 & 1454209 & 1423447 & 2897759 & 555017 & 5250 & 1806 \\
\hline f8 & 597560 & 786359 & 1268110 & 1633467 & 1740786 & 630153 & 6070 & 1826 \\
\hline f9 & 1022169 & 809340 & 1548441 & 1333164 & 1696100 & 926706 & 5155 & 2009 \\
\hline f10 & 5733.3502 & 15918.4959 & 7733.85187 & 15838.957 & 9506.22644 & 3768.21575 & 173.01441 & 84.914056 \\
\hline f11 & 4016.35353 & 12495.616 & 8658.49262 & 7811.13648 & 16183.5286 & 3276.58616 & 164.976562 & 76.1896413 \\
\hline f12 & 5581.02482 & 6369.15523 & 7799.50832 & 8543.755 & 9998.68019 & 4682.28745 & 208.685193 & 88.733396 \\
\hline f13 & 7115.33542 & 5571.35117 & 9378.1143 & 7938.1651 & 8617.41387 & 5837.59785 & 216.723343 & 142.895237 \\
\hline f14 & 43 & 105 & 49 & 248 & 98 & 13 & 2 & 9 \\
\hline f15 & 17 & 68 & 60 & 117 & 180 & 53 & 5 & 8 \\
\hline f16 & 30 & 27 & 60 & 130 & 144 & 54 & 5 & 1 \\
\hline $\mathbf{f 1 7}$ & 17 & 39 & 53 & 105 & 119 & 41 & 5 & 3 \\
\hline f18 & 95 & 349 & 209 & 433 & 267 & 114 & 5 & 1 \\
\hline f19 & 92 & 267 & 223 & 218 & 401 & 117 & 4 & 2 \\
\hline f20 & 101 & 124 & 198 & 252 & 243 & 103 & 4 & 4 \\
\hline f21 & 166 & 117 & 215 & 220 & 267 & 173 & 5 & 4 \\
\hline
\end{tabular}

\subsection{NMR response simulation by using ANN}

The proposed ANN approach was implemented on a PC with an Intel® Core $^{\mathrm{TM}}$ i3 $2.27 \mathrm{GHz}$ CPU, 4 GB RAM, the Windows 7 operating system. Using MATLAB® software, a Feed-Forward Back-propagation type network was designed within three layer for NMR Response estimation from micro-CT images, 
which is widely believed to outperform those with more layers (Balkin et al., 2000; Khan et al., 2008; Kadkhodaie-Ilkhchi et al., 2008; Kadkhodaie-Ilkhchi et al., 2010; Farzi, 2013; Bolandi et al., 2015). The network was then trained with the Levenberg Marquardt training algorithm (TrainLM), details of which is conveniently accessible in Bishop (1995) and Boadu (1997, 1998). Shifting from input layer to hidden layer is performed within Tangent logistic (TANSIG) transfer function whilst linear (PURLIN) transfer function preferentially connects the hidden and output layers. The input layer of the trained network consisted of 21 neurons, based on the 21 inputs (i.e. the number of extracted features), together with 14 neurons in the hidden layer. This was the optimal network setup as verified by the root mean squared error (RMSE) analysis, Fig. 4. Note that the network succeeded in minimizing the RMSE value in 11 epochs. Essentially, the performance of the model was measured by RMSE analysis; and this performance is equal to the expected value of the square of the difference between the value taken by the estimator and the parameter's true values, exploited to optimizing the value of weights and default bias. Thus, if a network runs multiple times, the optimum value of the parameters result in steady output with low uncertainty range. Alongside the performance, processing time is another issue which relevant to computational power. The architecture of the designed networks is demonstrated in Fig. 5.

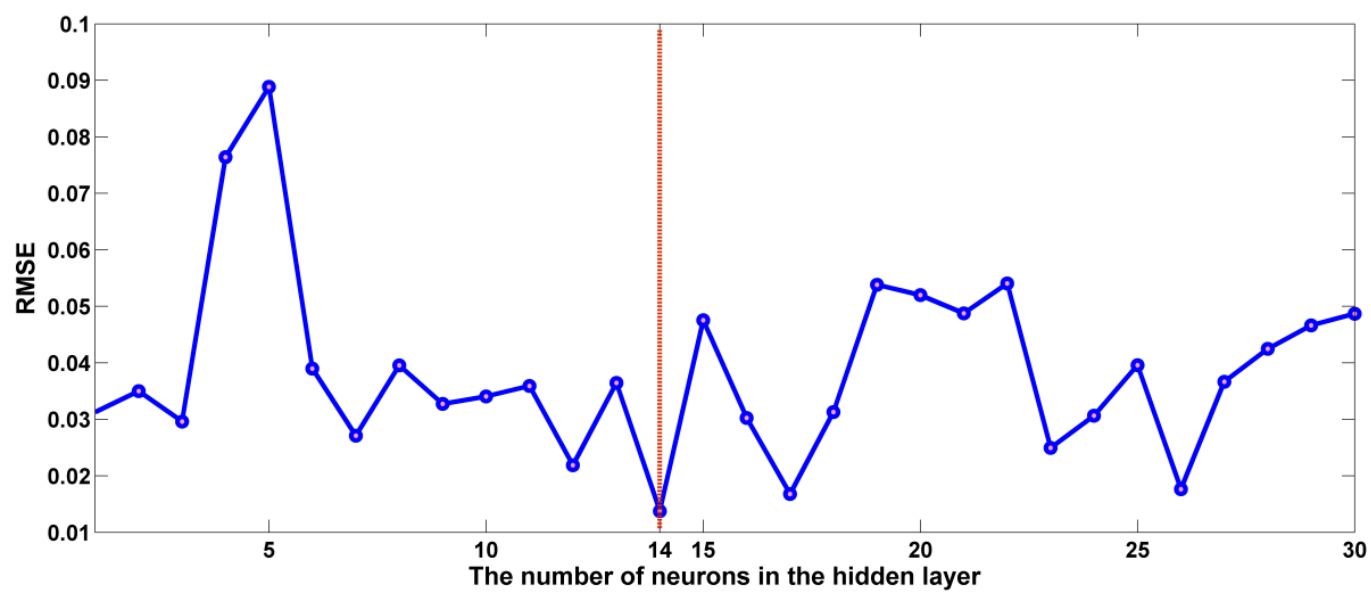

Fig. 4. Finding the optimum number of neurons via root mean square error (RMSE) analysis: the RMSE is plotted against the number of neurons in the hidden layer. 


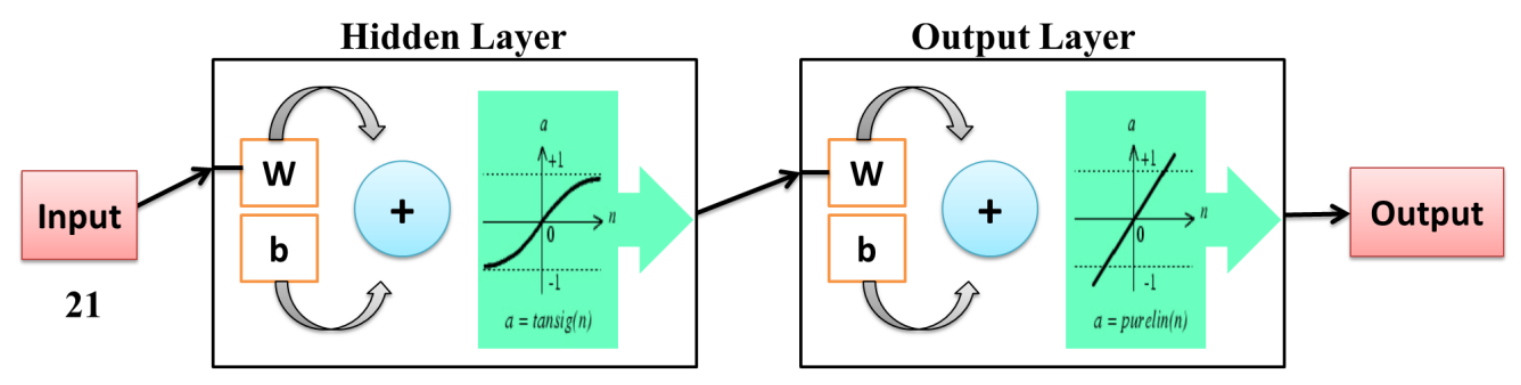

14

Fig. 5. Schematic diagram of constructed network with transfer functions. $w$ and $b$ are the weight and bias, respectively.

To predict the NMR parameters from image features using the ANN network, four sand packs (F42A, F42C, LV60A and LV60C), as well as two sandstones (Berea and S3) were selected. Two ANN models were constructed for estimation of $\mathrm{T}_{2}$ distribution and magnetization decay, in both models numerical image features were used as input values. In the final step, the ANN models were applied to simulating the NMR response of two additional sand packs (F42B and LV60B).

So far, Talabi (2008) simulated NMR responses using the RW method resulting in a good agreement between the simulated and the experimental measurements. This technique attempts to simulate $\mathrm{T}_{2}$ and magnetization decay by establishing a relationship between micro-CT images and NMR parameters. In this study, ANN systems were used to develop a new correlation for NMR parameter prediction based on micro-CT images. All the laboratory data set utilized for our analyses throughout this paper are taken from Talabi (2008) and Talabi et al. (2009). The comparisons between predicted and measured values utilizing RW and neural networks (Table 2) show both techniques were successful in generating the NMR parameters. Also, drawing an analogy between ANN and RW results shows that ANN has had an improvement on predicting the amplitude of the transverse magnetization and relaxation time. RMSE and correlation coefficient $(\mathrm{R})$ between measured and estimated data in test samples were considered as a criterion to evaluate the models accuracy. According to Fig. 6, the ANN simulated $\mathrm{T}_{2}$ curve and magnetization decay has approximately been fitted with experimental data. The ANN model predicted $\mathrm{T}_{2}$ 
curve for F42B and LV60B sand packs with RMSE of 0.0121 and 0.0037 and correlation coefficients; $\mathrm{R}^{2}$ $=0.9748$ and 0.9980 , respectively; which are the evidence of the best performance of the ANN model.

The ANN simulated magnetization decays and $\mathrm{T}_{2}$ distributions of the F42B and LV60B are compared with the experimental data and RW simulated curves in Fig. 7 and 8 . The results prove that ANN outperforms the RW method and it can be considered as a powerful tool for NMR response estimation, especially in cases where an accurate estimation criterion is critical.
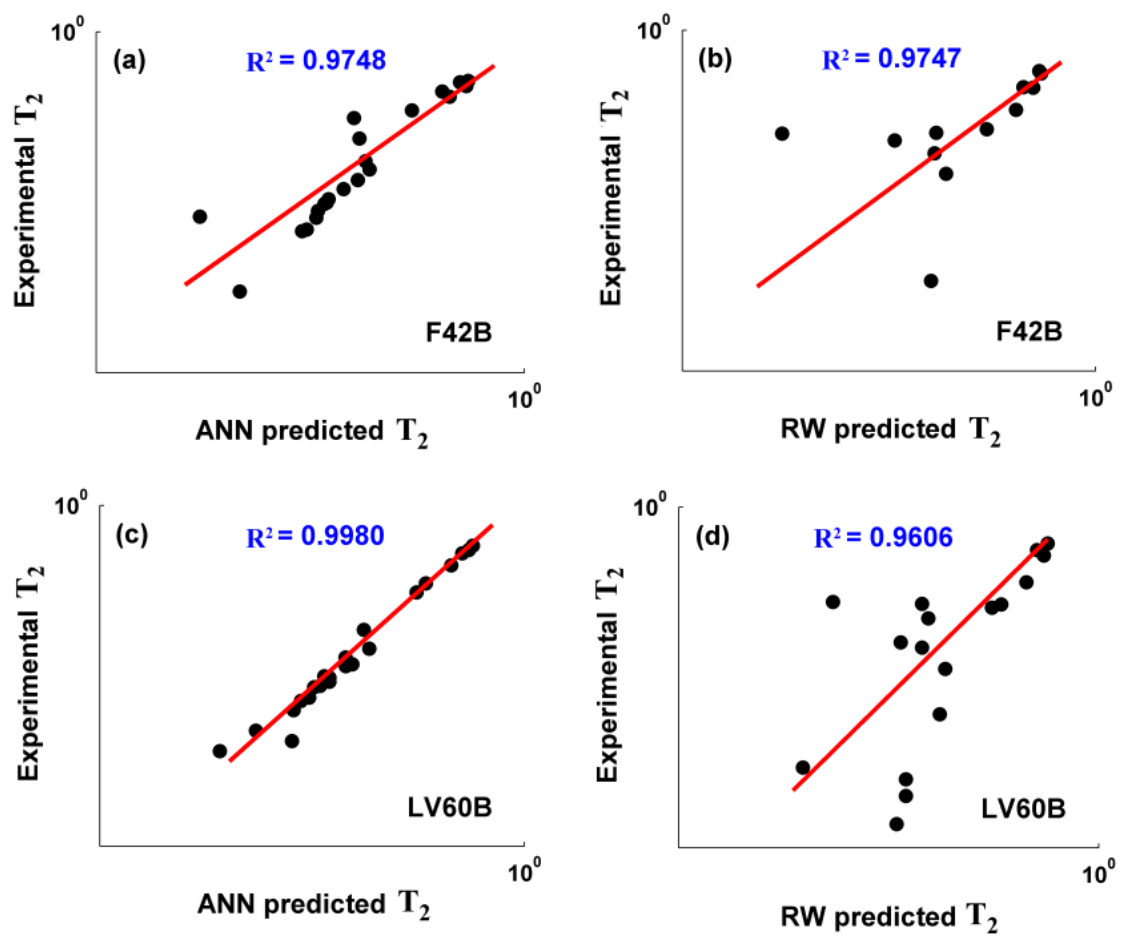

Fig. 6. Crossplots showing correlation coefficient between Experimental and predicted $\mathrm{T}_{2}$ distributions by ANN and RW for F42B and LV60B sand packs. 

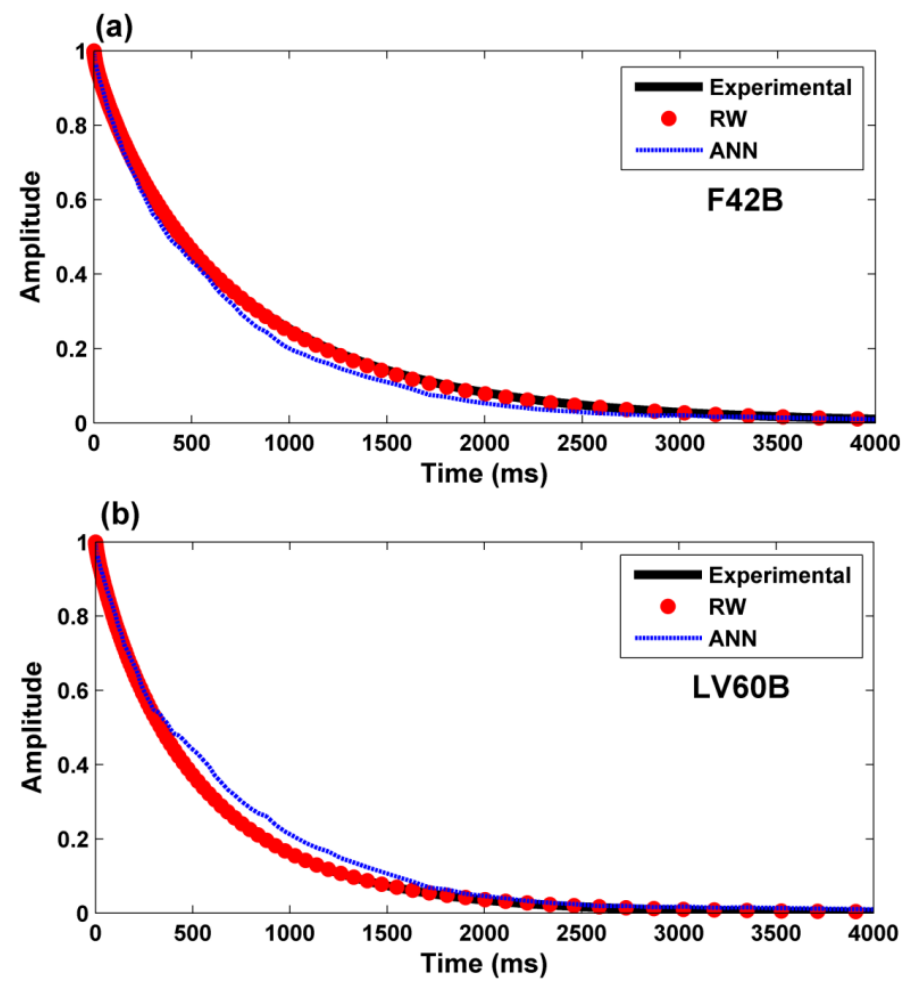

Fig. 7.a. Comparison of the experimental magnetization decay with RW and ANN predictions for F42B. b. Comparison of the experimental magnetization decay with RW and ANN predictions for LV60B.
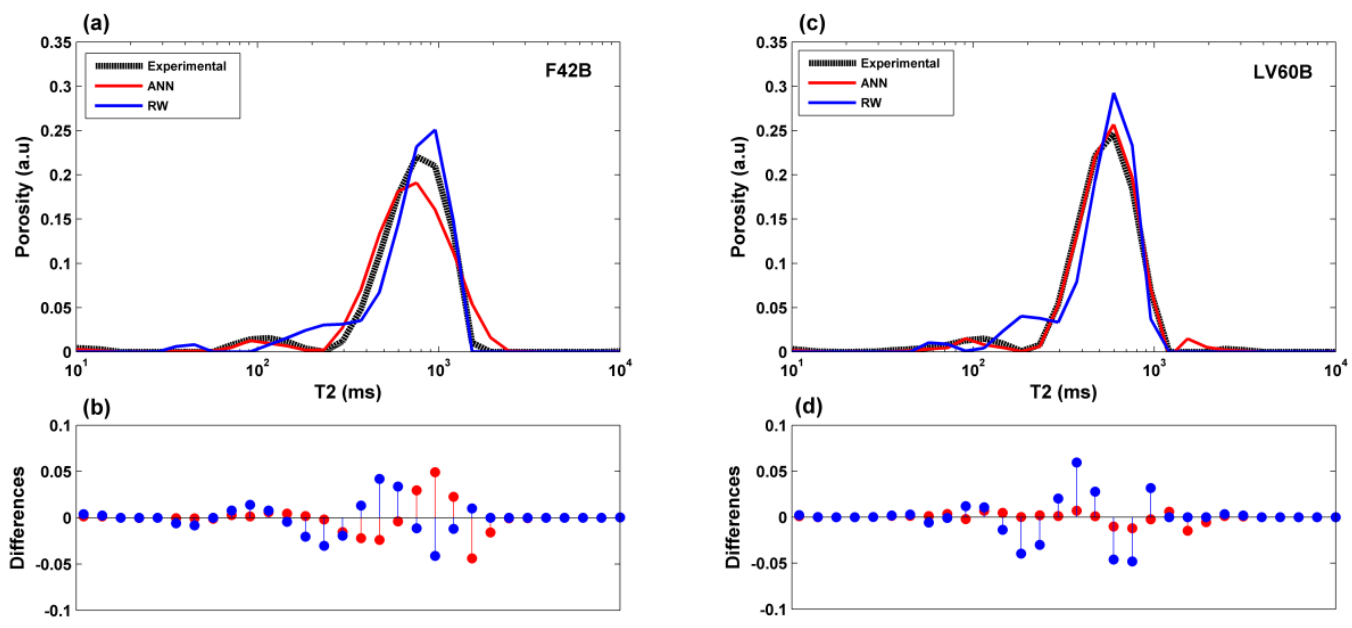

Fig. 8a. Comparison of the experimental $T_{2}$ distribution with RW and ANN predictions for F42B. b. Stem diagram of the differences in the porosity values of RW and ANN predictions with experimental T2 distribution for F42B. c. Comparison of the experimental T2 distribution with RW and ANN predictions for LV60B. d. Stem diagram of the differences in the porosity values of RW and ANN predictions with experimental T2 distribution for LV60B. 
Table 2. Comparison of RMSE for ANN and RW in predicting $\mathrm{T}_{2}$ distributions.

\begin{tabular}{ccc|c|c} 
& \multicolumn{2}{c|}{ ANN } & \multicolumn{2}{c}{ RW } \\
\cline { 2 - 5 } & RMSE & $\mathrm{R}^{2}$ & RMSE & $\mathrm{R}^{2}$ \\
\hline F42B & 0.0121 & 0.9748 & 0.0121 & 0.9747 \\
LV60B & 0.0037 & 0.9980 & 0.0162 & 0.9606 \\
\hline
\end{tabular}

\section{Conclusion}

The Nuclear Magnetic Resonance (NMR) response is one of the most noteworthy tools which is used to precisely characterize the reservoir without environmental effects. In the present study, we have predicted the NMR response from microCT numerical image features via an artificial intelligence algorithm with a good degree of accuracy. The micro-CT images of six sand packs were used for the construction of the neural network (ANN) model and two sand packs were taken to evaluate the satisfaction of the developed model. Accordingly, a Feed-Forward Back-propagation multilayered network took an input set of 21 numerical features predicted the $\mathrm{T}_{2}$ distribution together with the magnetization decay. Quantitative comparisons of the results and errors from ANN and RW predictions and experimental data demonstrate that for the estimation problem of $\mathrm{NMR} \mathrm{T}_{2}$ curve ANN readily outperforms RW in terms of accuracy $\left(\mathrm{R}^{2}\right.$ $\sim 0.98$ for the ANN model; while $\mathrm{R}^{2} \sim 0.97$ for the RW method on average). One of the other advantages of this method is that the solid phase was only considered and fluid phase does not play a role in this prediction technique. The main conclusion to be drawn is that the NMR response can be reliably and precisely simulated by using image features based neural network models.

\section{Acknowledgments}

The authors wish to acknowledge the Imperial College consortium on Pore-Scale Modeling for the preparation of the NMR measurements and their permission to use and publish the data. 


\section{References}

Asquith, G., Krygowski, D., 2004. Basic well log analysis. Second edition, AAPG, 245 p.

Balkin, S. D., Ord, K., 2000. Automatic neural network modeling for univariate time series,” International Journal of Forecasting, vol. 16 (4), pp. 509-515.

Bishop, C. M., 1995.Neural Networks for Pattern Recognition. Clarendon Press, Oxford. 670 pp.

Boadu, F.K., 1997. Rock properties and seismic attenuation: neural network analysis. Pure and Applied Geophysics 149, 507-524.

Blümich, B., 2005. Essential NMR for scientists and engineers. Springer-Verlag, Berlin Heidelberg, p. 243.

Boadu, F.K., 1998. Inversion of fracture density from field seismic velocities using artificial neural networks. Geophysics 63, 534-545.

Bolandi, V., Kadkhodaie-Ilkhchi, A., Alizadeh, B., Tahmorasi, J., Farzi, R., 2015. Source rock characterization of the Albian Kazhdumi formation by integrating well logs and geochemical data in the Azadegan oilfield, Abadan palin, SW Iran. Journal of Petroleum Science and Engineering, 133, 167-176.

Chen, S., Georgi, D., Fang, S., Salyer, J., Shorey, D., 1999. Optimization of NMR logging acquisition and processing, presented at SPE 74th Annual Conference and Technical Exhibition (ATCE), Houston, Texas. Coates, G. R., Xiao, L., Prammer, M.G., 1999. NMR logging: principles and applications. Halliburton energy services publication, $234 \mathrm{p}$.

Darling, T., 2005. Well logging and formation evaluation. Elsevier Inc, 326 p.

Farzi, R., 2012. Estimation of organic richness and geochemical zones using LogitBoosting and Fuzzy techniques. M.Sc. Thesis, Faculty of Geology, University of Tehran, Iran, pp 78.

Golsanami, N., Kadkhodaie-Ilkhchi, A., Sharghi, Y., Zeinali, M., 2014. Estimation NMR T 2 distribution data from well log data with use of a committee machine approach: A case study from the Asmari formation in the Zagros Basin, Iran. Journal of petroleum science and engineering, 114, pp 38-51. 
Heilbronner, R., Barrett, S., 2014. Image analysis in earth science: microstructures and textures of earth materials. Springer, $520 \mathrm{p}$.

Kadkhodaie-Ilkhchi, A., Rahimpour-Bonab, H., Rezaee, M. R., 2008. A committee machine with intelligent systems for estimation of total organic carbon content from petrophysical data: An example from the Kangan and Dalan reservoirs in south pars gas field, Iran. Computers \& Geosciences, 35, pp. 459-474.

Kadkhodaie-Ilkhchi, A., Monteiro, S.T., Ramos, F., Hatherly, P., 2010. Rock Recognition From MWD Data: A Comparative Study of Boosting, Neural Networks, and Fuzzy Logic. IEEE geoscience and remote sensing letters, vol. 7, no 4, pp. 680-684.

Kenyon, W.E., 1997. Petrophysical principles of applications of NMR logging. Log Anal 38, 21-43.

Khan, A. U., Bandopadhyaya, T. K., Sharma, S., 2008. Genetic algorithm based backpropagation neural network performs better than backpropagation neural network in stock rates prediction, IJCSNS International Journal of Computer Science and Network Security, vol. 8 (7), pp. 162- 166.

Labani, M. M., Kadkhodaie-Ilkhchi, A., Salahshoor, K., 2010. Estimation of NMR log parameters from conventional well log data using a committee machine with intelligent systems: A case study from the Iranian part of the South Pars gas field, Persian Gulf Basin. Journal of Petroleum Science and Engineering 72, pp. $175-185$.

Lawler, G., Limic, V., 2010. Random walk: A modern introduction. Cambridge university press, 364 p. Liu-Yu, S., Thonnat, M., 1997. Description of object shapes by apparent boundary and convex hull. Pattern Recognition 26, pp. 107-195.

Marmo, R., Amodio, S., Tagliaferri, R., Ferreri, V., Longo, G., 2005. Textural identification of carbonate rock by image processing and neural network: Methodology proposal and examples. Computer \& Geoscience, 31, 649-659.

Matlab user's Guide 2012. Neural Network, Fuzzy Logic and Direct Search toolboxes, Matlab CD-ROM, by the Mathworks, Inc.

Maute, R. E., 1992, Electrical Logging: State-of-theArt: The Log Analyst, v. 33, 206-227. 
Mohaghegh, S., Richardson, M., Ameri, S., 2001. Use of intelligent systems in reservoir characterization via synthetic magnetic resonance logs. J. Pet. Sci. Eng. 29, 189-204.

Talabi, O., 2008. Pore-scale simulation of NMR response in porous media. Ph.D. thesis, The department of earth science and engineering, Imperial College London, $148 \mathrm{p}$.

Talabi, O., AlSayari, S., Iglauer, S., Blunt, M. J., 2009. Pore-scale simulation of NMR response. Journal of Petroleum Science and Engineering, 67, 168-178.

Toumelin, E., Torres-Verdin, C., Chen, S., 2003. Modeling of multiple echo-time NMR measurements for complex pore geometries and multiphase saturations, SPE Reservoir Evaluation and Engineering, pp. 234-243. 